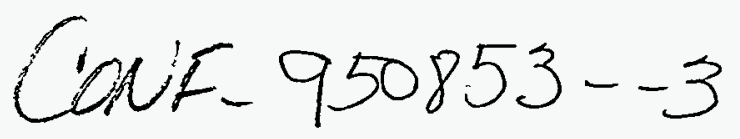

\title{
Numerical Predictions of the Separation of Heavy Components Inside the Trace Gas Concentrator ${ }^{1}$
}

\author{
J. D. Mo \\ Mechanical Engineering Department \\ The University of Memphis \\ Memphis, Tennessee \\ Andrew J. Szady \\ Martin Marietta Energy Systems, Inc. ${ }^{2}$ \\ Oak Ridge National Laboratory \\ P. O. Box 2003, Oak Ridge, Tennessee
}

\begin{abstract}
DISCLAIMER
This report was prepared as an account of work sponsored by an agency of the United States Government. Neither the United States Government nor any agency thereof, nor any of their employees, makes any warranty, express or implied, or assumes any legal liability or responsibility for the accuracy, completeness, or usefulness of any information, apparatus, product, or process disclosed, or represents that its use would not infringe privately owned rights. Reference herein to any specific commercial product, process, or service by trade name, trademark, manufacturer, or otherwise does not necessarily constitute or imply its endorsement, recommendation, or favoring by the United States Government or any agency thereof. The views and opinions of authors expressed herein do not necessarily state or reflect those of the United States Government or any agency thereof.
\end{abstract}

1. The submitted manuscript has been authored by a contractor of the U.S. Government under contract DE-AC05-84OR21400. Accordingly, the U.S. Government retains a paid-up, nonexclusive, irrevocable, worldwide license to publish or reproduce the published form of this contribution, prepare derivative works, distribute copies to the public, and perform publicly and display publicly, or allow others to do so, for U.S. Government purposes.

2. Managing contractor of the Oak Ridge K-25 Site, Oak Ridge Y-12 Plant, and Oak Ridge National Laboratory for the U.S. Department of Energy. 


\section{DISCLAIMER}

Portions of this document may be illegible in electronic image products. Images are produced from the best available original document. 


\title{
NUMERICAL PREDICTIONS OF THE SEPARATION OF HEAVY COMPONENTS INSIDE THE TRACE GAS CONCENTRATOR
}

\author{
J. D. Mo \\ Mechanical Engineering Department \\ The University of Memphis \\ Memphis, Tennessee \\ Andrew J. Szady \\ Oak Ridge National Laboratory \\ Oak Ridge, Tennessee
}

\begin{abstract}
- The component with a heavier molecular weight can be separated from the one with a lighter molecular weight in a binary mixture by applying an appropriate pressure gradient. A centrifugal force field effectively generates the required pressure gradient and a favorable flow field along the radial direction in a trace gas concentrator for such an application. This paper presents the numerical predictions of the mass separation inside a trace gas concentrator, which enriches Xenon in air. A NavierStokes solver in primitive variables using a pressure based algorithm has been applied to solve for the flow fields. Subsequently, the transport equations with a strong centrifugal field are solved for the mass concentration. This study is the continued effort for the proof-of-concept of centrifugal separation of components with a considerable difference in their molecular weight in a binary mixture. The significant effects of rotational speed, flow field, and the geometrical configuration on the mass separation are presented in this paper.
\end{abstract}

\section{INTRODUCTION}

The theoretical expression for the mass diffusion flux in a multicomponent system consists of the contributions of ordinary (concentration) diffusion, pressure diffusion and thermal diffusion (Bird, 1960). The ordinary diffusion contribution depends on the concentration gradients of all the substances present and has a tendency of unifying the mass distribution. Pressure diffusion indicates that there may be a net movement of the ith species in a mixture if there is a pressure gradient imposed on the system. The tendency for a mixture to separate under pressure gradient is very small, but use is made of this effect in component separations in which tremendous pressure gradients may be applied. A very high speed centrifuge provides the unique solution of creating high pressure gradients for separating one particular component from one with significantly different molecular weight in a binary mixture. The thermal diffusion describes the tendency for species to diffuse under the influence of a temperature gradient. This effect is also quite small, but, devices can be arranged to produce very steep temperature gradients so that separations of mixtures are effected.

It is the goal of the present work to explore the potential applications of the pressure diffusion mechanism of the separation of heavys from air in a trace gas concentrator. The mass transfer depends on both convection and diffusion. The convective mass transfer is a result of the flows of the mixture. The previous study on the flow characteristics has motivated a further exploration of the mass separation by the diffusion mechanism in a rotating system (Mo and Szady, 1995). The objectives of this study are twofold: 1) to develop the numerical model, and 2) to generate a data base using computational tools that provide guidance for the hardware design and performance analysis of a trace gas concentrator. It should be mentioned that only ordinary and pressure diffusion are considered in the diffusion mass transfer mechanism in the present work.

\section{PHYSICAL MODEL AND CONFIGURATION}

In the applications of a rotating system, the elementary and common configuration is cylindrical. The hardware geometry designed and developed by Oak Ridge National Laboratory is used for the computer model (Mo, 1994). A co-axial cylindrical centrifugal air concentrator rotating about the center axis, as 
illustrated in Fig.1, is the subject of this paper. The concentrator has a length of $0.32(\mathrm{~m})$, the rotor wall a radius of $0.1016(\mathrm{~m})$ and the center shaft a radius of $0.0833(\mathrm{~m})$. The simplified inlet and depleted outlet have an annular width of $0.928 \times 10^{-3}(\mathrm{~m})$. The annular width of the enriched stream outlet is $0.638 \times 10^{-5}$ $(m)$. Both the inlet and the depleted outlet are located on the center shaft. The inlet is $0.0833(\mathrm{~m})$ away from the bottom rotating end cap, and the depleted outlet is 0.0833 (m) away from the top rotating end cap. The remaining dimensions are given in Fig. 1. The whole rotor is enclosed in a vacuum casing in order to reduce the air drag when in rotation. The rotor can be rotated at various speeds by a drive motor installed on the rotating center shaft. The size of the casing is not pertinent in the present work.

The working fluid is the mixture of air and Xenon which is fed into a high-speed centrifuge concentrator. The mixture is modeled mathematically as a binary system, i.e. air and Xenon. Xenon has a molecular weight of 131 and is referred to as the heavy in this work. Air has an averaged molecular weight of 29 and is referred to as the lighter. The performance referred in this paper is the ratio of mole fraction at the cnriched outlet to the inlet mole fraction of the heavys. Physically, the lighter air flows from the inlet to the depleted outlet along the center shaft, but the heavys will have a higher concentration in the vicinity of the rotor wall under a large pressure gradient, as sketched in Fig.1. Therefore, how efficiently the heavys are collected depends on the rotational speed, the resulting flow characteristics, and other factors including the geometrical configuration and the size of the inlet and outlets, the total mass flow rate, the cut (which is defined as the percentage of the depleted flow rate over the total mass flow rate). This paper presents the numerical findings of the model and provides the proof-of-concept of the trace gas concentrator to some extent.

The basic flow conditions designated for the hardware are: the feed stream has a total flow rate of $2 \mathrm{~g} / \mathrm{s}$ at a pressure of 1.053 (ATM); the cut is $98 \%$, that means the enriched stream has a flow rate of $0.04 \mathrm{~g} / \mathrm{s}$ and the depleted stream has a flow rate of $1.96 \mathrm{~g} / \mathrm{s}$; at the inlet, the mole fraction of Xenon is $2.417 \times 10^{-8}$; and all the concentrator walls assume a constant temperature of $5599^{\circ}$. The rotational speed of the concentrator is $587.43 \mathrm{rev} / \mathrm{s}$.

The computer model presented in this paper assumes that the continuum equations for mass, momentum, and energy are sufficient to describe the flow. Based on the above described geometrical configurations and flow conditions, it is assumed that a stcady axisymmetrical swirling flow pattern is established in the concentrator. Thus, all the derivatives in the azimuthal $(\theta)$ direction may be omitted when the conservation equations are formulated in cylindrical coordinates, even though the defect velocity component in the radial direction is non-zero. Because of the high rotational speed, the pressure variation from the center shatt wall to the rotor wall is considerable based on the wheel flow solution. The wheel flow assumes that the fluid has a solid rotation. The mixture under study is treated as a perfect gas. The closure of the mathematical formulation is implemented by applying the ideal gas equation of state. Therefore, the density of the mixture changes significantly, and consequently the mixture pertains to be compressible. However, it is assumed that the compressible fluid properties such as dynamic and bulk viscosity, specific heat, thermal conductivity, and the molecular mass diffusivity are constant since the flow field is almost isothermal. It is also assumed that the change of the fluid property due to average molecular weight caused by the changes in concentration of small amounts of the heavys in the working fluid which is mostly air can be neglected. This allows the flow fields to be calculated separately from the calculation of the mass concentration.

Currently, only the steady state flow field is considered. For all the flow conditions investigated, the Reynolds number based on averaged velocity at the inlet and center shaft diameter is in the order of $10^{3}$, which indicates that it is reasonable to assume a laminar flow if the inlet flow keeps a lower level of turbulence. However, the high rotational motion of the fluid is a source of turbulence. Thus, a laminar model, but with an artificial viscosity, has been developed in the present study. Stability issues of this rotating flow are beyond the scope of this research.

\section{COMPUTER MODEL AND NUMERICAL METHOD}

\subsection{Hydrodynamic Model:}

The conservation equations for mass, momentum, and energy form the cornerstone of theoretical fluid dynamics. These equations describe the variations of fluid pressure, temperature, density, velocity, and other flow field properties throughout space and time. For the viscous compressible fluid flow in the present study, the basic equations employed are axisymmetric, multi-component conservation equations. A generalized form of these equations written in curvilinear coordinates is given by (Mo, 1994):

$$
\frac{1}{J} \frac{\partial \rho q}{\partial t}=\frac{\partial}{\partial \xi_{i}}\left[-\rho U_{i} q+\mu_{e} G_{i j} \frac{\partial q}{\partial \xi_{j}}\right]+S_{q}(1)
$$

where $q=1, u, v, w, h, k$, and $\varepsilon$ for the continuity, momentum, energy equations, and turbulence modeling, respectively. $J, U_{i}$ and $G_{i j}$ represent the Jacobian of the coordinate transformation, contravariant velocities, and diffusion metrics, respectively. $\mu_{\mathrm{e}}$ is the effective viscosity when the turbulent eddy viscosity concept is employed to model the turbulent flows. $S_{q}$ are the source terms. The equation of state for an ideal gas is used in the present study to close the above system of equations. The system of governing equations is formulated as a general timedependent form. Therefore, transient as well as steady state flow solutions can be determined by this time-dependent formulation.

The flow field under study is three-dimensional in general and dominated by the rotating motion component of the fluid even at the lowest rotational speed considered in this work (the range of the interested rotation speeds is from $5.87 \mathrm{rev} / \mathrm{s}$ to $587.43 \mathrm{rev} / \mathrm{s}$ ). The balance between the pressure gradient and the centrifugal force, with the corrections of viscous action on the Coriolis force, emerges as the backbone of the entire subject (Greenspan, 1968). The magnitude of the characteristic velocity in swirling is much higher than one in the axial plane, which 
creates enormous difficulty in numerical solution of the coupled fluid dynamic equations. In this work, rotating coordinates have been introduced in the numerical solution of these equations. Therefore, the magnitude of the swirling velocity component in the rotating system is compatible with the velocity components in other directions.

\subsection{Mass Transport Equations:}

The conventional discussion regarding mass fluxes and concentration gradients have been somewhat oversimplified. It is well known that momentum flux depends only on velocity gradient; energy flux depends on both temperature gradient and mechanical driving forces. However, mass flux depends on mechanical driving forces, which include ordinary, pressure, and forced diffusion, as well as temperature gradient (soret effect). As far as the present work is concerned, the mass flux equation for a binary system with species A and B can be reduced to the following form:

$$
\begin{aligned}
& J_{B}^{(x)}=\frac{C^{2}}{\rho R T} M_{A} M_{B} D_{A B} x_{A} \frac{\partial G_{A}}{\partial x_{B}} \nabla x_{B} \\
& J_{B}^{(p)}=\frac{C^{2}}{\rho R T} M_{A} M_{B} D_{A B} x_{A}\left[\frac{\bar{V}}{M_{A}}-\frac{1}{\rho}\right] \nabla p
\end{aligned}
$$

Thermal and force diffusion are not discussed here.

Therefore, the total mass flux for species B become

$$
\begin{aligned}
& J_{B}=J_{B}^{(x)}+J_{B}^{(\rho)} \\
& =\frac{C^{2}}{\rho R T} M_{A}^{2} M_{B} D_{A B} x_{A}\left(\frac{1}{M_{A}} \frac{\partial G_{A}}{\partial x_{B}} \nabla x_{B}+\left[\frac{\vec{V}}{M_{A}}-\frac{1}{\rho}\right] \nabla p\right)
\end{aligned}
$$

and for species $\mathrm{A}$ in the binary system

$$
\begin{aligned}
& J_{A}=-J_{\dot{B}} \\
& =-\frac{C^{2}}{\rho R T} M_{A}^{2} M_{B} D_{A B} x_{A}\left(\frac{1}{M_{A}} \frac{\partial G_{A}}{\partial x_{B}} \nabla x_{B}+\left[\frac{\bar{V}}{M_{A}}-\frac{1}{\rho}\right] \nabla p\right)
\end{aligned}
$$

by using thermodynamic relationships like

$$
\left(d \bar{G}_{A}\right)_{T, p}=R T d \ln a_{A}
$$

in which a $A$ is the activity. Then

$$
J_{A}=-\frac{C^{2}}{\rho} M_{A} M_{B} D_{A B}\left(\frac{\partial \mathrm{n} a_{A}}{\partial x_{A}} \nabla x_{A}+\frac{M_{A} x_{A}}{R T}\left[\frac{\bar{V}}{M_{A}}-\frac{1}{\rho}\right] \nabla p\right)
$$

in which the first part represents ordinary diffusion and the second part represents pressure diffusion. For an ideal solution, activity is proportional to mole fraction.
Finally, the mass equation for component $A$ is:

$$
\begin{aligned}
& \frac{\partial}{\partial}\left(\frac{\rho}{M} x_{A}\right)+\nabla \cdot\left(\frac{\rho}{M} \bar{u} x_{A}\right) \\
& =\nabla \cdot\left\{\frac{\rho}{M} \frac{M_{B}}{M} D_{A B}\left(\frac{\partial n a_{A}}{\partial x_{A}} \nabla x_{A}+\frac{\left(1-x_{A}\right) x_{A}\left(M_{B}-M_{A}\right)}{\rho R T}\right) \nabla p\right\}
\end{aligned}
$$

\section{$\underline{3.3 \text { Grid Generation }}$}

To be consistent with the general form of the governing equation formulation, which is accurate and efficient in the numerical solution of these equations, a body-fitting orthogonal grid system is employed. This grid system is provided by an algebraic grid generator which has been implemented into this computer model(Mo and Chow, 1993). Through the specification of proper parameters, a favorable grid network can be obtained that allocates more grid points at the flow inlet and outlets. The grid is also denser toward the center shaft and rotor walls, where flow variables have large gradients.

\subsection{Numerical Scheme}

A pressure based solution method was selected for this work. To solve the system of nonlinear coupled partial differential equations (1), finite difference approximations are used to establish a system of linearized algebraic equations. A relaxation solution procedure is employed to couple the goveming equations. A simplified momentum equation is combined with the continuity equation to form a pressure correction equation. The pressure correction equation exhibits elliptic behavior for this low speed flow. To solve the system of linear algebraic equations, a semi-implicit matrix solver is utilized. This matrix solution algorithm is a modified Stone's method, which has been demonstrated to be very efficient for matrix systems resulting from complex curvilinear coordinates, including high grid aspect ratios and high grid skewness. However, this method does require a large amount of memory.

\subsection{Boundary and Initial Conditions}

The flow fields are represented in a computational domain: that is, each point in the physical domain is represented by a point in computational space. Boundary conditions are assigned to each of the physical boundaries and are represented in computational space. The velocity and pressure are specified on the inlet plane. The flow variables at the exit are determined by extrapolating from upstream values, except for the mass flow rates which are fixed as given in above sections. All wall surfaces of the concentrator are modeled as no-slip, isothermal. Pressure over the wall surface is extrapolated from the grid points interior to the surface. A zero solution (on the interior grid points, velocity is zero) is used to start the numerical modeling. 


\section{RESULTS AND DISCUSSIONS}

The computer modeling was conducted on An IRIS INDIGO R4000 workstation located at the University of Memphis. From the grid sensitivity study, it was concluded that a grid density of $181 \times 12$ is sufficient and that an increased grid density did not significantly alter the results. Therefore, all the results presented in this paper were obtained from the grid density of 181x121. Fig. 2 shows the computational grid network having a total of 21901 grid points. The convergence of the solution is monitored using a global residual of the conservation variables. Each case takes about 40 hours CPU on the above mentioned workstation.

In this study, one major series of results are presented to illustrate the effects of the depleted outlet location on the resulting flow fields, and subsequently on the mass separation. Some light is also shed on the impact of the diffusivity. Convincing experimental evidence shows that rotating flows have a strong disposition towards two-dimensionality-even in distinctively non-linear circumstances (Greenspan, 1968). In addition, an extrapolation of the stability analysis results for finite length rotating annulus flow indicates that the flows under study fall in the lower symmetric regime (Zheng, Mo and Antar, 1995). As a result, it is assumed that a steady state of the flow field can be achieved for the present application. The numerical results obtained in this work have confirmed this conclusion to some extent.

As discussed in the previous paper (Mo and Szady, 1995), a random streamline pattern appears as the rotational speed increases to some critical value. These random cells (or noise) are believed to be attributed to turbulence. To remove the noise in the solution, an artificial turbulence model has been used betore actual turbulence modeling is introduced. The so-called artificial model is constructed by simply increasing the laminar viscosity to the turbulent viscosity level for fluid dynamics solutions. The reason for using an artificial viscosity model is for the numerical efficiency, since turbulence modeling is very timeconsuming. The model generates reasonable results.

The mass transfer is attributed to the convection and diffusion in general. Conceptually, the trace gas concentrator makes use of the pressure gradient to separate the components with significantly different molecular weight in a mixture along the radial direction. This mechanism is made use of under the assumption that there is no strong convection in the radial direction. Otherwise, the net mass movement due to the pressure gradient will be easily smoothed out by the convection. Whether this flow field assumption is realistic or not in the concentrator chamber is the first concern of the present work. Fig. 3 shows the computed flow field at the designated mass flow rate and cut as well as rotational speed. It is clear that the noise in the solution has been effectively removed. In the solution, the magnitude of the artificial viscosity is adjusted to have an oscillation-free solution. The viscosity used for all the results presented in this section is 100 times the molecular viscosity, which is evidenced to be in the same order as the turbulent viscosity from the turbulence modeling. Looking into the flow field, it can be seen that the flow field has become favorable for the mass separation as described earlier. The main stream is packed to the center shaft with very weak recirculation in the outermost part of the chamber. Fig. 4 shows the pressure contours. These contours and their distribution are very close to the wheel flow results. Fig. 5 shows the Xenon concentration contour plot. The radial front of the mass separation can be clearly observed. The noticeable distortion is a result of the flow recirculations. From these results, it can be seen how important the flow field is. Fig. 6(a) and (b) show the Xenon concentration distribution along the rotor wall and the center shaft wall, respectively. It can be seen that downstream from the inlet, along the center shaft wall, the Xenon concentration becomes lower and lower. This is because the pressure-induced diffusion normal to the stream direction makes a net mass movement of Xenon away from the center shaft wall. However, Xenon concentration increases when getting close to the depleted outlet. This phenomenon is due to convection, which brings the higher Xenon concentration fluid to the outlet from the interior domain where the Xenon mass concentration is higher. The Xenon concentration distribution along the rotor wall exhibits a peak. Looking into the flow field, it is found that this concentration peak on the rotor is exactly located at the flow stagnation point resulting from recirculations. How to capture this peak point for the best output of the enriched outlet needs further exploration. However, there is a strong possibility that a peak of the heavys concentration on the rotor is necessary to maintain the enriched output.

Fig. 7 shows the computed flow result of the case when the depleted outlet is located at the middle point of the center shaft. The pattern of the flow field is similar to the case presented above. The only exception is that the main recirculation is shorter in the axial direction. The feature of the mass separation is similar, as well. Fig. 8 shows the concentration distribution along the side walls. Again, it can be seen that there is a peak point in the concentration which is higher than the value at the enriched outlet, but the location of the peak point is different from the previous case. The other locations of the depleted outlet have also been studied. The modeled results, including flow field characteristics and the gas separation performance for different cases, are all similar. But, the configuration referred to as the designated case generates the best output in terms of the mole fraction ratio at the enriched outlet. This conclusion is consistent with the engineering model as well as with the physical explanation. The physical explanation is the longer the distance between the inlet and the depleted outlet is, the longcr time of mass separating the fluid elements experience. It should be pointed out that all the mass separation performance are predicted under molecular diffusivity. The effective diffusivity in a turbulent flow is much higher than molecular one, which will be the subject of future work.

Obviously, the artificial model is not physical. However, these simple models provide quick information to guide the comprehensive model. As a result of the above solutions, the turbulence modeling will only concentrate on the designated configuration. 


\section{CONCLUSION}

The computational tools developed in this study provide reasonable predictions of the flow field as well as mass separation information inside the trace gas concentrator. The results obtained from this computational study indicate that:

1. Centrifugal air concentrator generates mass separation and the separation ratio is about 2.0 under the rotation rate considered and molecular diffusivity, which means the enriched stream contains double mass concentration of the heavys than at the inlet. This mass separation efficiency is interesting enough to justify further study.

2. Radial velocity component is quickly stagnated by the rotational cffects. Consequently, the main stream is packed close to the center shaft and a very weak flow field appears in the outer part of the concentrator chamber. This flow characteristic prevents strong convection from the mass transfer.

3. The swirling dominates the flow field, so that the pressure and density field are only slightly disturbed from the equilibrium state of the wheel flow. This wheel flow pressure gradient produces the net mass movement.

4. The study shows that the farther the depleted outlet is located from the inlet, the more favorable the flow field becomes for the mass separation. It is reasoned that the farther distance provides longer time for fluid element to experience mass diflusion.

\section{ACKNOWLEDGMENT}

Kesearch performed under Subcontract SR301-85-RC, with the University of Memphis under Martin Marietta Energy Systems, Inc., contract DE-AC05-84OR21400 with the U. S. Department of Energy.

\section{REFERENCES}

Bird, R. B., Stewart, W. E. and Lightfoot, E. N., 1960, Transport Phenomena.

Mo, J., 1994, "Computer Model of Centrifugal Air Concentrator," Final Report 85X-SR301V, Oak Ridge National Laboratory.

Mo, J. and Szady, A., 1985, "Numerical Modeling of the Flow Field inside the Centrifugal Air Concentrator," AIAA Paper 95-0701, Reno, Nevada.

Mo, J. and Chow, A., 1993, "A Generic Adaptive Grid Generator in FDNS Code," 11th CFD Workshop of NASA, Huntsville, Alabama

Greenspan, H. P.,1968, The Theory of Rotating Fluids, Cambridge at the University Press.

Olander, D. R., 1978, "The Gas Centrifuge," Scientific American, Vol. 239, No. 2.

Wood, H. and Morton, J. 1980, "Onsager's Pancake Approximation for the Fluid Dynamics of a Gas Centrifuge," J. Fluid Mech., Vol. 101.
Zheng, Y. M., Mo, J. D. and Antar, B. N., 1995, "A Numerical Study of Strongly Nonlinear Baroclinic Instability," AIAA Paper 95-0157, Reno, Nevada.

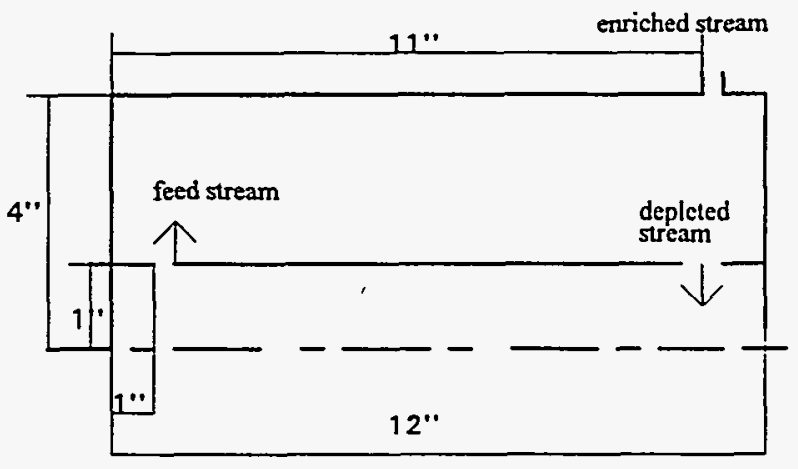

Fig.1 Configuration of geometry and flow information

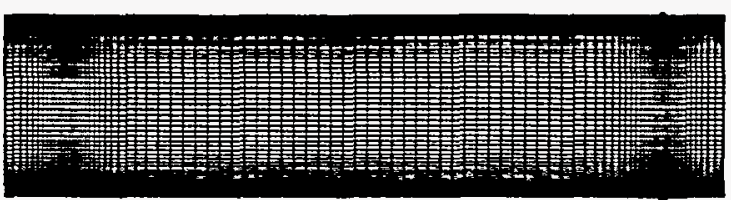

Fig. 2. Sample grid network.

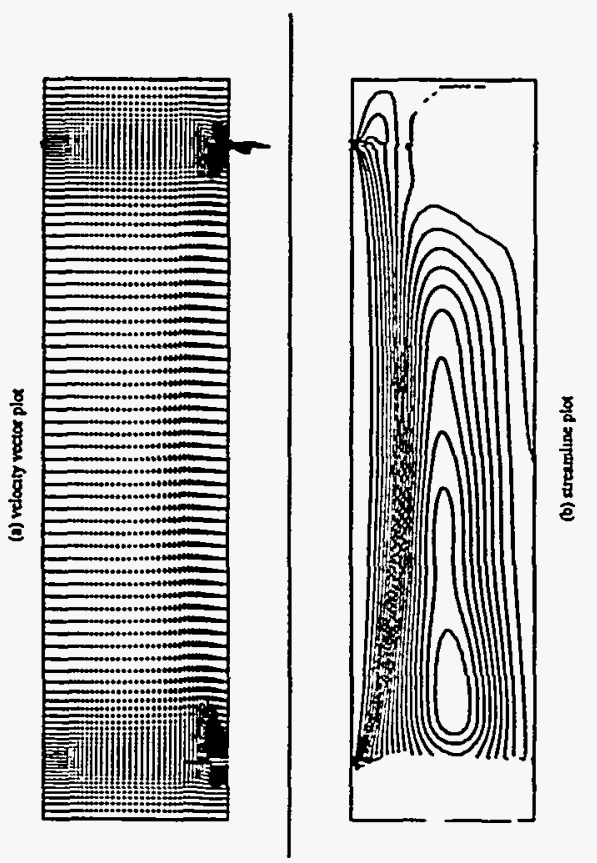

Fig. 3. Computed flow field for the depleted outlet 1 " from the top cap. 


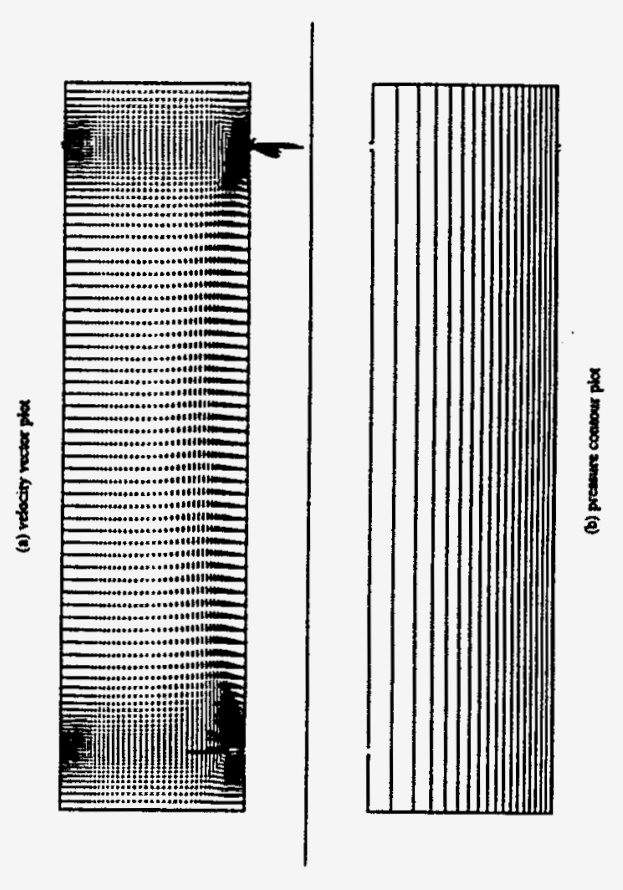

Fig. 4. Pressure distribution for the depleted outlet 1 " from the top cap

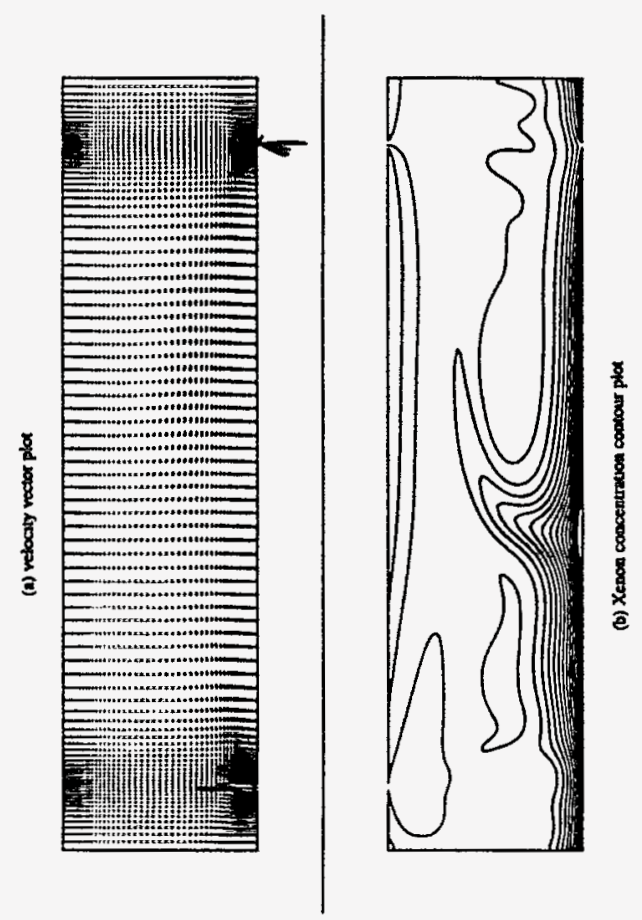

Fig. 5. Xenon concentration contour plot for the depleted outlet 1 " from the top cap
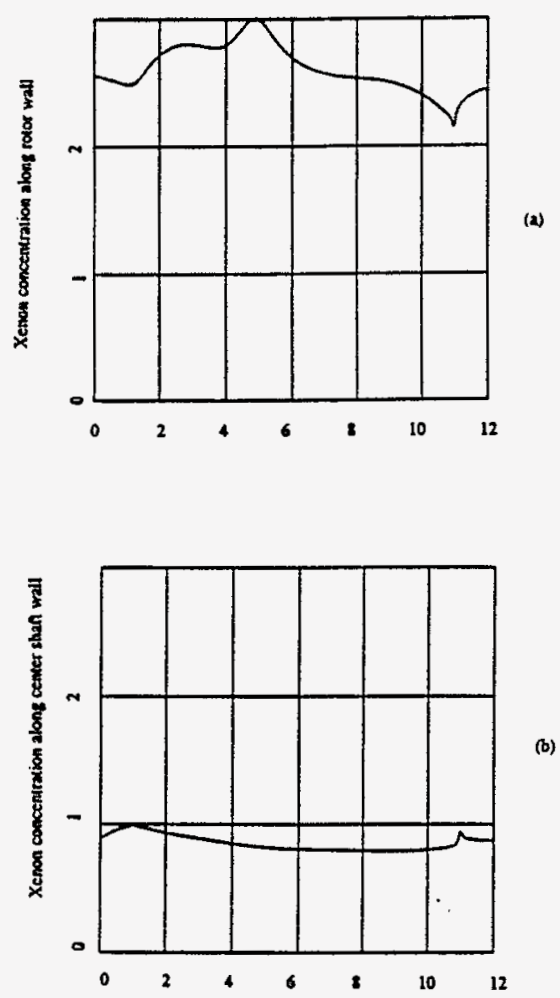

Fig. 6. Wall Xenon concentration distribution for the depleted outlet 1 " from the top cap

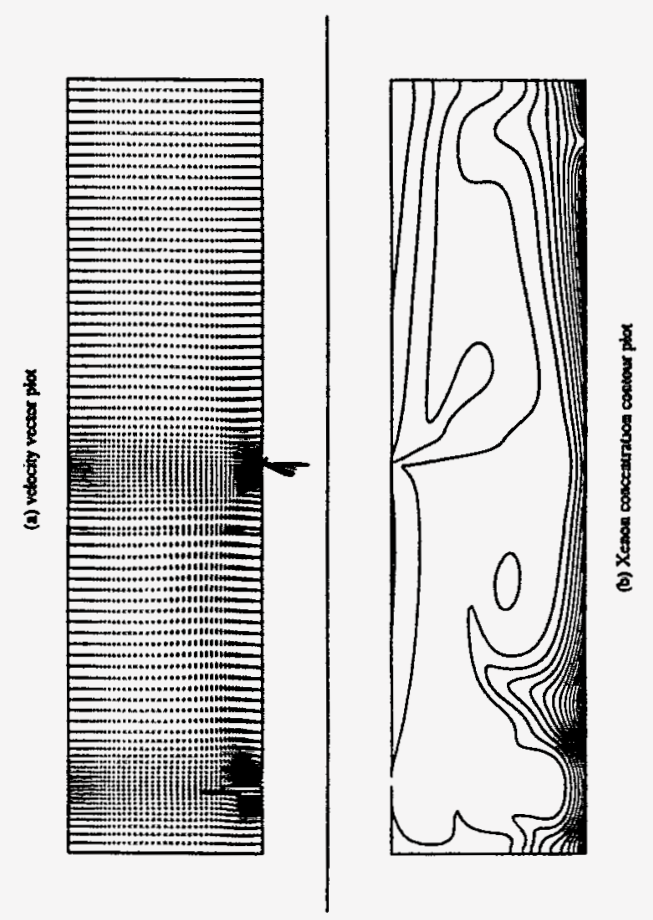

Fig. 7. Computed flow field and the Xenon concentration for the depieted outlet 6" from the top cap 

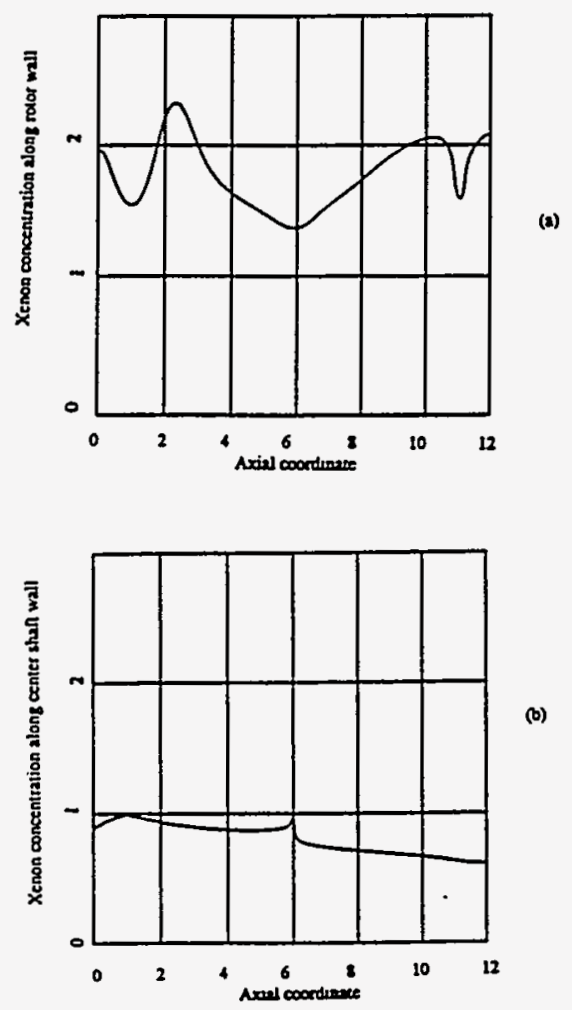

Fig. 8. Wall Xenon concentration distribution for the depleted outlet 6" from the top cap 